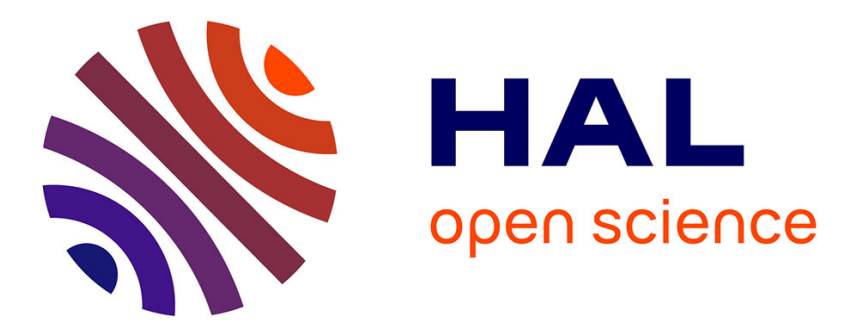

\title{
Temperature dependent photoluminescence of anatase and rutile TiO 2 single crystals: Polaron and self-trapped exciton formation
}

Mathieu Gallart, Thomas Cottineau, Bernd Hönerlage, Valérie Keller, Nicolas Keller, Pierre Gilliot

\section{To cite this version:}

Mathieu Gallart, Thomas Cottineau, Bernd Hönerlage, Valérie Keller, Nicolas Keller, et al.. Temperature dependent photoluminescence of anatase and rutile TiO 2 single crystals: Polaron and self-trapped exciton formation. Journal of Applied Physics, 2018, 124 (13), pp.133104. 10.1063/1.5043144 . hal02384105

\section{HAL Id: hal-02384105 \\ https://hal.science/hal-02384105}

Submitted on 28 Nov 2019

HAL is a multi-disciplinary open access archive for the deposit and dissemination of scientific research documents, whether they are published or not. The documents may come from teaching and research institutions in France or abroad, or from public or private research centers.
L'archive ouverte pluridisciplinaire HAL, est destinée au dépôt et à la diffusion de documents scientifiques de niveau recherche, publiés ou non, émanant des établissements d'enseignement et de recherche français ou étrangers, des laboratoires publics ou privés. 


\section{Temperature dependent photoluminescence of anatase and rutile $\mathrm{TiO}_{2}$ single crystals: Polaron and self-trapped exciton formation}

Mathieu Gallart, Thomas Cottineau, Bernd Hönerlage, Valérie Keller, Nicolas Keller, and Pierre Gilliot

Citation: Journal of Applied Physics 124, 133104 (2018); doi: 10.1063/1.5043144

View online: https://doi.org/10.1063/1.5043144

View Table of Contents: http://aip.scitation.org/toc/jap/124/13

Published by the American Institute of Physics

\section{Articles you may be interested in}

Electrical properties of bulk semi-insulating $\beta-\mathrm{Ga}_{2} \mathrm{O}_{3}(\mathrm{Fe})$

Applied Physics Letters 113, 142102 (2018); 10.1063/1.5051986

Theoretical confirmation of the polaron model for the $\mathrm{Mg}$ acceptor in $\beta-\mathrm{Ga}_{2} \mathrm{O}_{3}$

Journal of Applied Physics 124, 145702 (2018); 10.1063/1.5049861

Donors and deep acceptors in $\beta-\mathrm{Ga}_{2} \mathrm{O}_{3}$

Applied Physics Letters 113, 062101 (2018); 10.1063/1.5034474

Effect of boron addition in modulating the optoelectronic properties of undoped and Al-doped $\mathrm{ZnO}$ thin films Journal of Applied Physics 124, 135103 (2018); 10.1063/1.5046188

Defects responsible for charge carrier removal and correlation with deep level introduction in irradiated $\beta_{-\mathrm{Ga}_{2} \mathrm{O}_{3}}$ Applied Physics Letters 113, 092102 (2018); 10.1063/1.5049130

Acceptor doping of $\beta-\mathrm{Ga}_{2} \mathrm{O}_{3}$ by $\mathrm{Mg}$ and $\mathrm{N}$ ion implantations Applied Physics Letters 113, 102103 (2018); 10.1063/1.5050040

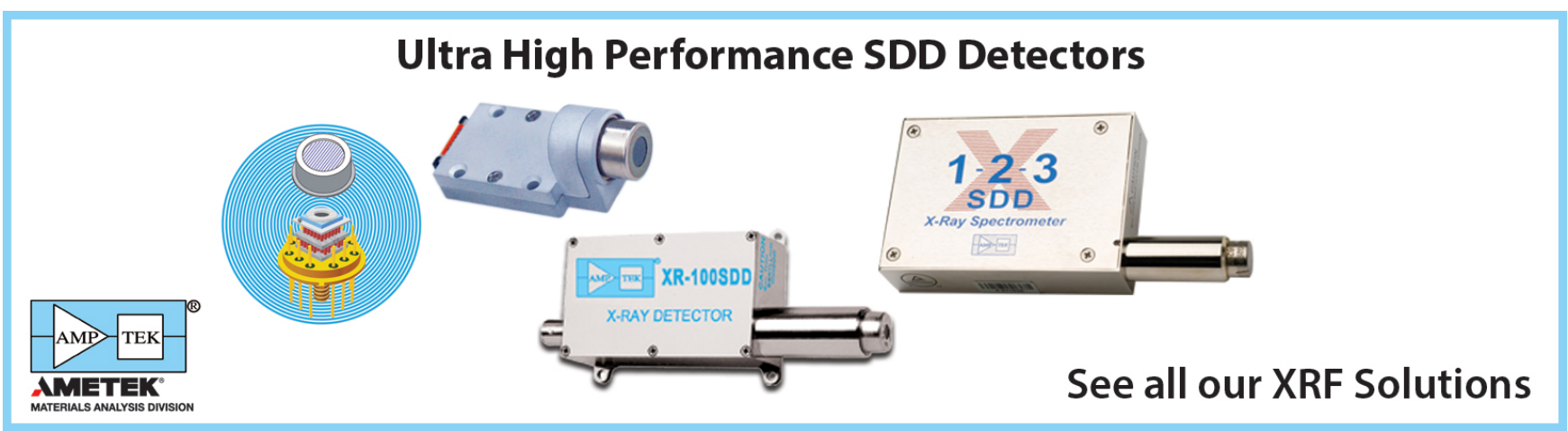




\title{
Temperature dependent photoluminescence of anatase and rutile $\mathrm{TiO}_{2}$ single crystals: Polaron and self-trapped exciton formation
}

\author{
Mathieu Gallart, ${ }^{1, a)}$ Thomas Cottineau, ${ }^{2}$ Bernd Hönerlage, ${ }^{1}$ Valérie Keller, ${ }^{2}$ Nicolas Keller, ${ }^{2}$ \\ and Pierre Gilliot ${ }^{1}$ \\ ${ }^{1}$ Institut de physique et chimie des matériaux de Strasbourg, UMR 7504 CNRS-université de Strasbourg, \\ 23 rue du Loess, BP 43, F-67034 Strasbourg, France \\ ${ }^{2}$ Institut de chimie et procédés pour l'énergie l'environnement et la santé, UMR 7515 CNRS-université de \\ Strasbourg, 25 rue Becquerel, F-67087 Strasbourg, France
}

(Received 6 June 2018; accepted 4 September 2018; published online 5 October 2018)

\begin{abstract}
We propose an analysis of the emission properties of anatase and rutile titanium dioxide $\left(\mathrm{TiO}_{2}\right)$ that emphasizes the role of the strong electron-phonon interaction. We performed measurements of photoluminescence (PL) spectra of bulk monocrystals under continuous wave-laser excitation and of their temperature dependence. We show that in both anatase and rutile, weakly bound self-trapped excitons are actually made out from carrier polarons and give rise to a broad emission band in the visible spectral range. The thermal activation of carrier motion allows their hopping to distant sites that leads to the observed quenching of luminescence. In the specific case of rutile $\mathrm{TiO}_{2}$, the PL spectral shape and its intensity-quenching scenario reveal the presence of dark trap states. Moreover, an additional narrow line structure shows up at low temperatures. The latter is due to localized impurity states that can be attributed to oxygen vacancies and can be fitted with a large Huang-Rhys parameter $S=2.5$ within a Franck-Condon model. Both phases show thus a very strong interaction between the photogenerated carriers and the lattice. Published by AIP Publishing.

https://doi.org/10.1063/1.5043144
\end{abstract}

\section{INTRODUCTION}

Because of its high quantum yield and a suitable electronic band structure, titanium dioxide $\left(\mathrm{TiO}_{2}\right)$ is a very promising metal oxide semi-conducting material ${ }^{1}$ for photo-conversion applications such as photocatalytic reactions and as transport material in photovoltaic cells [dye-sensitized solar cells $(\mathrm{DSSC})^{2}$ and perovskite solar cells]. It is not expensive, non-toxic, and resistant towards photo-corrosion in a wide range of $\mathrm{pH}$ values. Rutile and anatase phases are the two main crystalline polymorphs of $\mathrm{TiO}_{2}$, constituted of chains of $\mathrm{TiO}_{6}^{2-}$ octahedrons, sharing edge and summit ${ }^{3}$ in which a $\mathrm{Ti}^{4+}$ ion is surrounded by $6 \mathrm{O}^{2-}$ ions. Both crystal structures are stable under ambient conditions, but differ by a distortion of the octahedrons and by their different arrangements in the crystals. ${ }^{4,5}$ Anatase $\mathrm{TiO}_{2}$ has a band gap of about $3.31 \mathrm{eV}$, and that of rutile $\mathrm{TiO}_{2}$ is about $3.02 \mathrm{eV}$ at $77 \mathrm{~K}^{6-11}$ While both phases may be photoactivated by UV light, the anatase form is supposed to be a more photocatalytic active phase than the rutile one due to a higher Fermi energy and a higher surface hydroxylation rate.

Photocatalytic and more globally photo-conversion applications imply the use of nanoparticles $(\sim 10-20 \mathrm{~nm})$ in order to have an important surface to volume ratio and due to the limited diffusion length of holes. In photocatalytic applications, two factors are very important: first, the system should have a low band-gap energy and, second, fast electron-hole recombination in the $\mathrm{TiO}_{2}$ volume or at the $\mathrm{TiO}_{2} /$ adsorbate interface should be prevented. In order to initiate redox

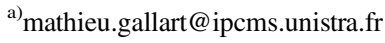

reactions with adsorbed surface species in photocatalysis or to collect the maximum number of carriers in photovoltaic devices, one should have a high density of free carriers. This can be achieved by optical excitation of electron-hole pairs. The recombination of photo-generated electron-hole pairs reduces, however, the density of available charge carriers at the active particle surface. In addition, it was observed that not only the size of the $\mathrm{TiO}_{2}$ particles but also their surface properties, crystalline structure, or degree of crystallization affect the photocatalytic efficiency. ${ }^{12-14}$

Although titania nanoparticles have been already extensively studied, the intrinsic processes underlying the behavior of the photoexcited charge carriers, including their relaxation, transport, and recombination, are not fully understood and still need to be resolved. ${ }^{15,16}$

From many studies performed on the electronic and optical properties on $\mathrm{TiO}_{2}$, some experimental facts and some typical features have been evidenced and are the basis of our understanding: First, the absorption of titanium dioxide occurs in the near UV, while the observed photoluminescence (PL) shows a very large Stokes shift with a very broad emission band centered in the visible spectral range. Second, a very efficient charge separation allows the collection of electrons and holes or their trapping at different locations that could be on the surface in the case of nanocrystals. Third, a strong electron-phonon coupling leads to lattice deformations that give rise to the formation of polarons ${ }^{17,18}$ and self-trapped excitons (STE). ${ }^{19-21}$ Fourth, oxygen vacancies can be numerous, with concentration in rutile as high as $0.5 \%\left(3 \times 10^{20} \mathrm{~cm}^{-3}\right){ }^{22}$ allowing an efficient trapping of carriers or polarons.

Nevertheless, a comprehensive picture of the carrier behavior, that could unify all the experimental observations 
by involving these mechanisms, is still missing. This concerns even the PL origin for which a long time ago several hypotheses were published together with the first studies of light emission in $\mathrm{TiO}_{2}$. Since that time, a large set of different interpretations is still proposed and used as described in recent review papers. ${ }^{23-26}$

Our study aims at giving some insight into the processes that the carriers come across after their excitation and at clarifying which mechanism is dominant. We start for that purpose from the observations and interpretations of various processes that can be found in the literature. We complement them by using an experimental method that we have successfully applied previously to nanocrystals, ${ }^{6,12,27}$ where we measure the decrease of the photoluminescence intensity with temperature, from helium up to room temperature. The competition between the carrier separation and their recombination, and specifically their radiative recombination, makes PL experiments a method of choice for determining the scenario followed by the carriers after their photo-creation. Their radiative recombination needs an overlap of their wave functions, and observing a PL emission is indeed a good indication that electron and hole remain close together. Following the PL efficiency as a function of temperature gives, moreover, a good insight into the processes that could be thermally activated and that could give rise to the carrier transfer towards distant sites. In addition, the study of PL temperature dependence is a sensitive and powerful technique to characterize defect states in semiconductors. ${ }^{28,29}$ The temperature dependent PL gives thus information on the recombination and relaxation scenario of photo-induced electron-hole pairs as well as on the energy of defect states that could be possibly involved in the process. ${ }^{6,11}$

With respect to our previous works, ${ }^{6,12,27}$ we choose here to study the nature of the electronic states and the carrier recombination scenario in bulk crystals of anatase and rutile $\mathrm{TiO}_{2}$ in order to get information on the intrinsic processes that can depend on the crystal phase (anatase or rutile). We aim here at disentangling as far as possible the intrinsic properties of the material, due to the crystal structures, from extrinsic processes that are related to quantum size, geometry, or surface effects. Even if, as reported in the work of Monticone et al. ${ }^{30}$ no quantum size effect is observed for $\mathrm{TiO}_{2}$ particles with a diameter above $1.5 \mathrm{~nm}$, surface effects remain important for larger nanocrystals. As a first necessary step before further studying more complex nanostructured $\mathrm{TiO}_{2}$ architectures with enhanced photoactivity, we will thus, in the following, base our analysis on PL experiments performed on a set of bulk samples where carriers can evolve in the crystal volume. That will give valuable information even for particles or layers of nanometric sizes where the optical, electrical, and photo-catalytic properties are highly influenced by the band structure inherited from the bulk crystals.

In the following, the description of our experiments and the fit of our data will be used to establish a scenario, which is apt to explain the observed properties of $\mathrm{TiO}_{2}$ : We will start from an analysis of the spectral shape and position of the photoluminescence. In order to explain their properties, we propose a separate interaction of electrons and holes with the lattice. Thus, polarons are formed, which interact only weakly with one another. They can be considered as loosely bound, self-trapped excitons. This clarifies the picture in which selftrapped excitons are involved and which is usually invoked to explain the large Stoke shift of the luminescence.

The observed behavior of the luminescence emission intensity as a function of temperature is then explained as a thermally activated motion of those polarons that can reach distant sites. Since the wave-functions of electron- and hole polarons no longer overlap, they cannot radiatively recombine, leading to a decrease of photoluminescence intensity with increasing temperature.

Finally, we explain the different temperature behaviors of the photoluminescence intensity in the rutile phase when compared to anatase in detail and evidence again the strong vibrational coupling of trapped carriers. This set of points shows that, among various effects involving surface states or different types of defects extensively discussed in the literature, the coupling with lattice vibration is to our notion of predominant importance in $\mathrm{TiO}_{2}$. It is so strong that it is entangled in relaxation and in emission processes for both free and trapped electronic excitations in $\mathrm{TiO}_{2}$.

\section{EXPERIMENTAL SETUPS}

\section{A. Anatase and rutile $\mathrm{TiO}_{2}$ single crystal samples}

The samples studied here are commercial single crystals: 3 rutile samples (MTI Corporation, USA) with (100), (001), and (110) orientated top surfaces (labeled R100, R001, and R110 in the following) and 3 anatase samples (SPL, The Netherlands) with (100), (001), and (110) orientated surfaces (labeled A100, A001, and A110, respectively).

\section{B. Temperature dependent photoluminescence measurements}

The photoluminescence (PL) of the samples was measured as a function of temperature by mounting the samples in a liquid helium bath Oxford cryostat in which the temperature is stabilized and can be continuously varied between $4 \mathrm{~K}$ and $300 \mathrm{~K}$.

The samples were excited with continuous wave $(\mathrm{CW})$ Melles-Griot $\mathrm{HeCd}$ laser UV light at $325 \mathrm{~nm}(3.814 \mathrm{eV})$ at low intensity. The PL emission of the samples was spectrally resolved by a SPEX $270 \mathrm{M}$ spectrograph and registered by a Roper liquid nitrogen cooled CCD camera. The excitation intensity was kept low in our experiments (less than $2 \mathrm{~W} /$ $\mathrm{cm}^{2}$ ) since the spectral PL emission shape of the rutile samples is known to change drastically under high excitation conditions, ${ }^{15,16}$ indicating that new recombination channels are opened due to electron-hole collision processes.

Photoluminescence excitation (PLE) experiments were also performed. In PLE experiments, the emission efficiency is determined by a three-step process: light absorption inducing transition toward an excited level, non-radiative relaxation between the excited level and the emitting level, followed by the radiative emission. The PL emission was measured at the emission maximum as a function of the excitation wavelength in the $300-500 \mathrm{~nm}$ range with a spectral 
resolution of $1 \mathrm{~nm}$. The PLE spectra, plotted as a function of excitation photon energy, allow one to measure indirectly the sample absorption spectra. We used light emitted by a broad-spectrum Energetiq ${ }^{\circledR}$ EQ-99FC laser-driven light source (LDLSTM), which was spectrally filtered by a SPEX $270 \mathrm{M}$ spectrograph used as a monochromator before exciting the samples.

\section{Emission and absorption of anatase and rutile $\mathrm{TiO}_{2}$}

Figure 1 shows as an example the PLE spectra of the samples A001 at $44 \mathrm{~K}$ and R110 at $8 \mathrm{~K}$. The PLE spectra of both, rutile and anatase types, samples show a steep increase when increasing the excitation photon energy, followed by a slower, monotonous decrease at higher photon energies. The absorption edges can be determined from the low photon energy inset at $411 \mathrm{~nm}(3.016 \mathrm{eV})$ for the rutile and around $377 \mathrm{~nm}(3.288 \mathrm{eV})$ for the anatase sample, which are in close agreement with literature values. ${ }^{1}$ Similar to the results discussed in Ref. 1, the anatase $\mathrm{TiO}_{2}$ samples showed a PL in the visible spectral range centered at $560 \mathrm{~nm}(2.21 \mathrm{eV})$ with a FWHM of about $600 \mathrm{meV}$, while that of the rutile $\mathrm{TiO}_{2}$ was centered in the infrared at $840 \mathrm{~nm}(1.48 \mathrm{eV})$ with a FWHM of about $150 \mathrm{meV}$.

PLE and PL spectra show thus a very large Stokes shift of the emission with respect to the absorption. Moreover, the observed emissions are spectrally broad (Fig. 1) and do not appear as emission peaks but as broad bands.

\section{TEMPERATURE DEPENDENT PHOTOLUMINESCENCE OF ANATASE $\mathrm{TIO}_{2}$ SAMPLES}

Let us first describe the PL intensity temperature dependence of the anatase samples, on which we will base our analysis of their emission properties. According to Fig. 1, the PL maximum is centered on $2.2 \mathrm{eV}(560 \mathrm{~nm})$ and depends only slightly on the sample. Between $10 \mathrm{~K}$ and 70 $\mathrm{K}$, the PL maximum position is almost fixed for all three samples, but it changes between $80 \mathrm{~K}$ and $200 \mathrm{~K}$. This variation is not monotonous but shows a minimum around $150 \mathrm{~K}$,

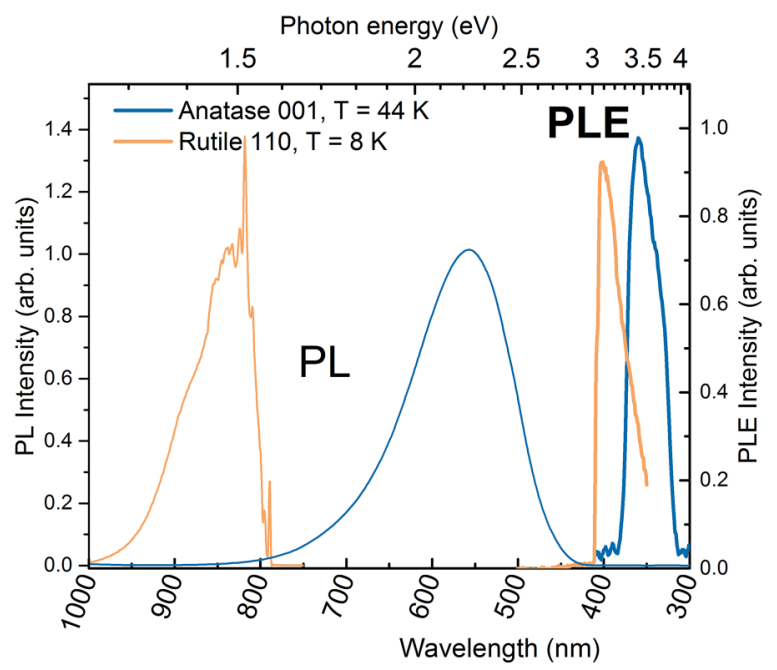

FIG. 1. Photoluminescence excitation (PLE) spectra and photoluminescence (PL) spectra of samples A001 at $44 \mathrm{~K}$ and R110 at $8 \mathrm{~K}$. which is most pronounced in sample A001 and the least in sample A110.

As shown by Tang et al., ${ }^{34}$ this behavior observed on the line maximum spectral position when increasing the temperature is typical for samples that contain aluminum. They observed a red-shift of the lines with increasing temperature in aluminum doped samples while a blue shift occurs in undoped samples. We have performed scanning electron microscopy measurements on our samples, which confirm the presence of aluminum, but the corresponding experimental uncertainty does not allow to improve the analysis already developed by Tang et al. and to determine the impurity concentrations in our samples precisely. We do not attribute, however, the spectral shape of the PL on the orientation of the crystal surface that is different for our three samples, but on the different aluminum concentrations that they probably contain.

The observation of Tang et al. did not receive an explanation and our measurement can hardly give it. One can nevertheless remark that the red shift with increasing temperature observed in our sample occurs on a limited spectral range of $150 \mathrm{meV}$, while Tang et al. ${ }^{34}$ measured a shift as large as $550 \mathrm{meV}$ for an aluminum concentration of $300 \mathrm{ppm}$. The samples that we studied are thus only slightly doped and will show features close to that of the undoped samples.

Figure 2 shows the PL intensity integrated over the whole emission band. It decreases in all three samples by about 2 orders of magnitude when increasing temperature from $10 \mathrm{~K}$ to $200 \mathrm{~K}$. Similar to the PL maximum position, the PL quenching with temperature is comparable for the three samples in the interval from $10 \mathrm{~K}$ to $70 \mathrm{~K}$. It becomes, however, sample dependent when heating from $80 \mathrm{~K}$ to $200 \mathrm{~K}$.

In order to analyze the PL temperature dependence, we assume a competition between radiative emission from the states, which give rise to the PL and the population transfer towards non-radiative states, which can be thermally activated. As it will be discussed further, when following the model described for nanocrystals, ${ }^{6,12,31}$ this leads to a quenching of the PL intensity with increasing temperature.

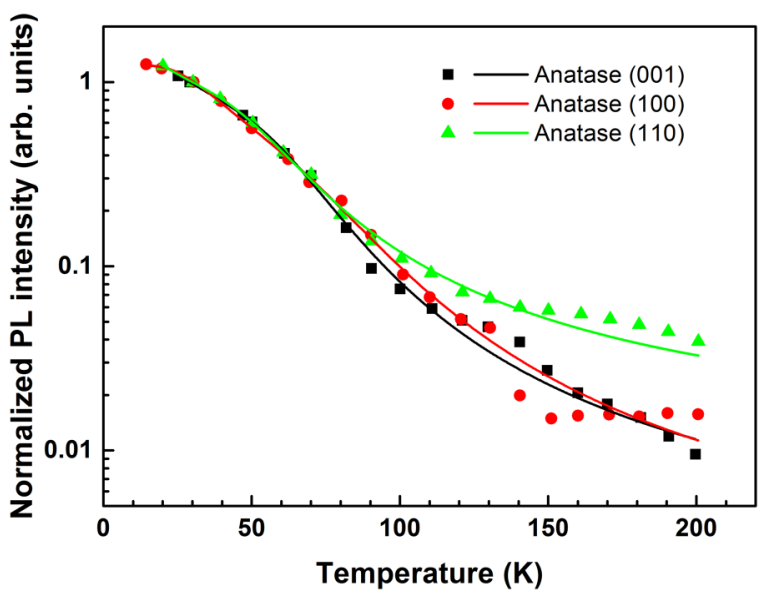

FIG. 2. Normalized integrated PL intensity as a function of temperature for the anatase samples A001, A100, and A110. Full lines: Adjustment with Eq. (1). See text and supplementary material SI1. 
Concerning our model, let $\mathrm{I}_{0}$ denote the PL emission intensity in the limit of low temperatures and $\mathrm{I}(\mathrm{T})$ this intensity as a function of the temperature. As previously discussed for anatase $\mathrm{TiO}_{2}$ nanocrystals, ${ }^{6,12}$ we find that at least two activation processes with different activation energies $E_{a}$ and $E_{b}$ have to be considered (Fig. 3) in our bulk anatase $\mathrm{TiO}_{2}$ in order to account for the experimental results. Indeed, if no second non-radiative recombination channel "b" (with an activation energy $E_{b}>E_{a}$ ) is considered, the thermal PL-quenching process is saturated in a very small temperature range. Now, the full thermal PL-quenching process can be modeled by a 4-level system. When introducing the energy differences $\Delta E_{i}=\left(E_{i}-E_{R}\right)$ between the radiative state " $R$ " and the two thermally activated states " $i=(a, b)$, the emission intensity $\mathrm{I}(\mathrm{T})$ is given by

$$
I(T)=\frac{I_{0}}{1+A_{a} \exp \left(\frac{-\Delta E_{a}}{k_{B} T}\right)+A_{b} \exp \left(\frac{-\Delta E_{b}}{k_{B} T}\right)},
$$

where $A_{a}=\tau_{R} /\left(\tau_{a}+\tau_{a \downarrow}\right)$ and $A_{b}=\tau_{R} /\left(\tau_{b}+\tau_{b \downarrow}\right)$ are coefficients given by the ratio of the luminescent radiative lifetime $\tau_{R}$ and the inverse transition probabilities of the non-luminescent states $i=(a, b)$ to the luminescent one $\tau_{i \downarrow}$ and to the ground state $\tau_{\mathrm{i}}$. The full model of this 4-level system is sketched in Fig. 3 and detailed in the supplementary material.

We use the formula of Eq. (1) in order to fit the data of Fig. 2. One should keep in mind that both activation levels only simulate the real situation, which is much more complex: the important spectral width of the PL corresponds to a broad inhomogeneous band indicating that neither the ground state nor the radiative state has a fixed energy but the convolution of their energy distributions gives rise to the observed PL spectral width of $600 \mathrm{meV}$ (FWHM). The spectral position of the a and b levels (Fig. 3) and the transfer rates should, as well, fluctuate. The numbers that we determine by our fits (Table I) are thus the mean values of dispersed data.

The fit results are shown in Fig. 2 by the full lines for the three anatase samples. The resulting parameter values of $\mathrm{A}_{\mathrm{i}}$ and $\Delta \mathrm{E}_{\mathrm{i}}$ are given in Table I. As stated above, all three samples show first (up to temperatures of about $70 \mathrm{~K}$ ) the same PL quenching behavior, which results in similar values of the activation energies $\Delta \mathrm{E}_{\mathrm{a}}$ and of the quenching amplitudes $A_{a}$. It is interesting to notice that in all cases, the radiative recombination times are always longer than the non-radiative recombination times, as shown by the

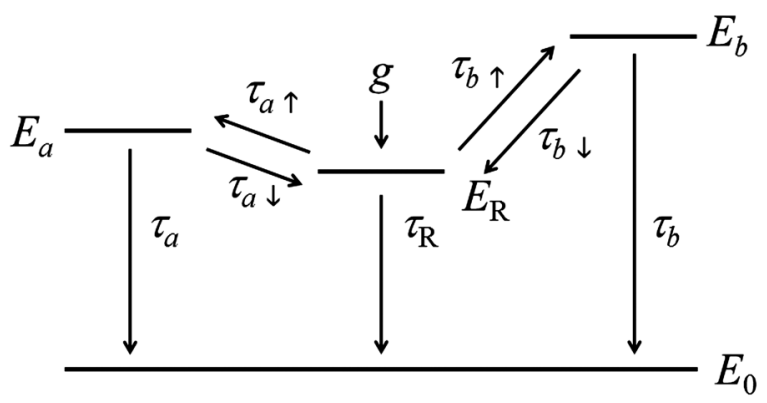

FIG. 3. 4-level system to simulate the PL quenching scenario with temperature in anatase titania. Details are given in supplementary material SI1. values of $\mathrm{A}_{\mathrm{a}}$ that depend on the ratio of these times. Taking into account the values of the activation energies $\Delta \mathrm{E}_{\mathrm{a}}$, this indicates that the quantum efficiency of the radiative recombination is quite low at room temperature when compared to the non-radiative one.

At higher temperatures, the activation energies $\Delta \mathrm{E}_{\mathrm{b}}$ and quenching amplitudes $A_{b}$ of samples A001 and A100 on the one side, and A110 on the other side are quite different, indicating changes of the non-radiative channels. It is interesting to notice that sample A110 shows almost a saturation behavior. This is due to the coefficient $A_{b}=\tau_{R} /\left(\tau_{b}+\tau_{b \downarrow}\right)$, which is much smaller in this sample than in the samples A001 and A100. Nevertheless, in all samples, we observe an activation energy $\Delta \mathrm{E}_{\mathrm{b}}$ that is nearly five times larger than $\Delta \mathrm{E}_{\mathrm{a}}$, while the order of magnitude of both activation energies is the same in all the samples. This shows that the same two processes are observed in all the samples. Time-resolved PL experiments performed on commercial powders using a setup based on a streak camera showed that the dynamics of photoexcited carriers lie in many temporal ranges, from ps up to $\mu \mathrm{s}$. This result is compatible with the dynamics that is suggested by our analysis of the static PL evolution with temperature, where the ratio between photoluminescence and trapping times given by the $A_{b}$ coefficient (Table I) can reach 3 orders of magnitude.

\section{TEMPERATURE DEPENDENT PHOTOLUMINESCENCE OF RUTILE $\mathrm{TiO}_{2}$ SAMPLES}

For rutile samples, Fig. 4(a) shows the PL spectrum of the sample R100 as a function of the temperature as an example. The other samples with the rutile structure show the same features. The PL spectrum is centered at $840 \mathrm{~nm}$ $(1.5 \mathrm{eV})$ with a FWHM of about $150 \mathrm{meV}$, i.e., the PL is emitted at much lower photon energies and its spectral width is much narrower than the PL of the anatase samples. In addition to a broad PL band, sharp lines show up in the spectra as shown in Fig. 4(b).

When increasing the temperature, the sharp lines broaden, decrease in intensity, and completely disappear around $130 \mathrm{~K}$. As discussed below in detail, they are tentatively attributed to the recombination of electron-hole pairs, bound to crystal imperfections. The intensity of the broad band, on the contrary, increases drastically when increasing the temperature from $4 \mathrm{~K}$ to $50 \mathrm{~K}$. This increase is followed by a PL quenching process at even higher temperatures. During heating, the spectral shape of the PL low energy side and the position of the PL maximum remain unchanged, while the high energy wing of the spectrum increases significantly.

TABLE I. Parameter values defined in Eq. (1) for the three anatase $\mathrm{TiO}_{2}$ bulk samples (A001, A100, and A110).

\begin{tabular}{lrrcc}
\hline \hline $\mathrm{TiO}_{2}$ sample & \multicolumn{5}{c}{$\begin{array}{c}\Delta \mathrm{E}_{\mathrm{a}} \\
(\mathrm{meV})\end{array}$} & \multicolumn{1}{c}{$\mathrm{A}_{\mathrm{b}}$} & $\begin{array}{c}\Delta \mathrm{E}_{\mathrm{b}} \\
(\mathrm{meV})\end{array}$ \\
\hline $\mathrm{A} 001$ & $5 \pm 1.7$ & $7 \pm 2.0$ & $915 \pm 260$ & $37 \pm 2.6$ \\
$\mathrm{~A} 110$ & $2.5 \pm 0.7$ & $4 \pm 1.9$ & $179 \pm 24$ & $25 \pm 1.6$ \\
$\mathrm{~A} 100$ & $14 \pm 2.5$ & $10.8 \pm 0.6$ & $1343 \pm 640$ & $45 \pm 4.4$ \\
\hline \hline
\end{tabular}




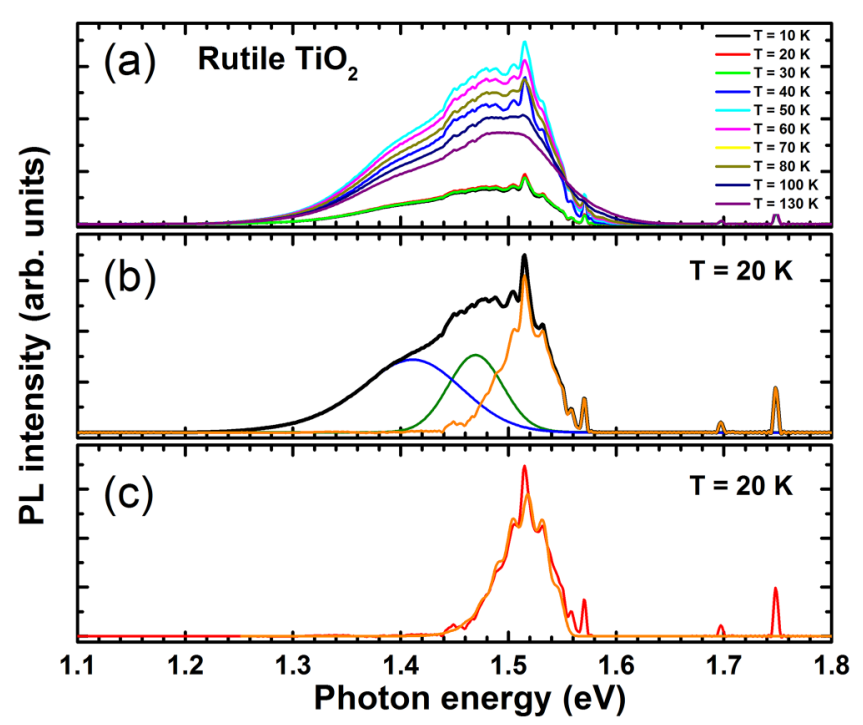

FIG. 4. (a) PL spectrum of the rutile sample R100 for different temperatures. (b) Analysis of the PL emission into two broad components and a set of narrow lines at higher energies. (c) Simulation of the structured high-energy part of the PL emission by a Franck-Condon emission spectrum.

Figure 5 shows the integrated PL intensity of the rutile samples as a function of temperature. The integrated PL intensity mainly characterizes the broad PL band since the integrated intensity of the narrow lines is smaller in comparison. When increasing temperature from $4 \mathrm{~K}$ to $30 \mathrm{~K}$, the integrated PL intensity remains first constant. Then, it increases strongly when rising the temperature to $50 \mathrm{~K}$. At even higher temperatures, between $50 \mathrm{~K}$ and $200 \mathrm{~K}$, the PL intensity decreases as observed in anatase samples. As shown in Fig. 5, this qualitative behavior is common to all three rutile samples, independent of their surface orientation. The PL quenching scenario is, however, slightly different.

In order to analyze the results of Fig. 5 in detail, we consider, similar to the scheme used for anatase samples, a multilevel model involving radiative and non-radiative states (Fig. 6). But we assume furthermore that, after their generation

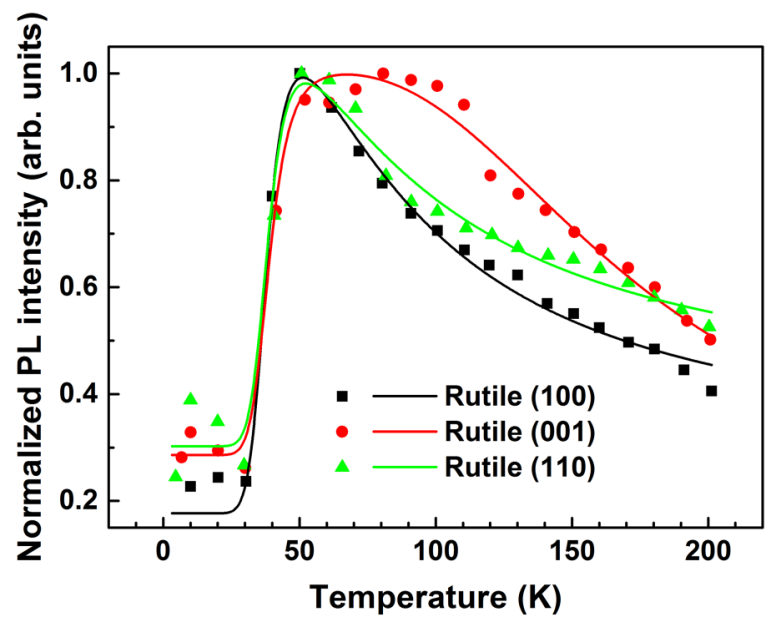

FIG. 5. Normalized integrated PL intensity as a function of the temperature for the rutile samples (R001, R100, and R110). Full lines: Adjustment with Eq. (2). See text and supplementary material SI2.

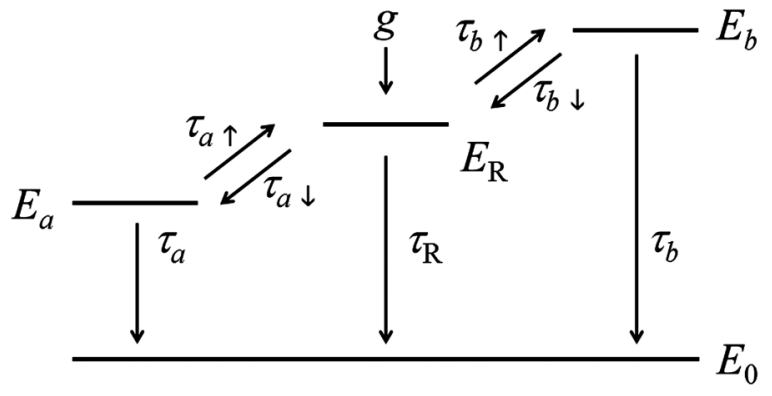

FIG. 6. 4-level system to simulate the PL quenching scenario with temperature in rutile titania. Details are given in supplementary material SI2.

by light absorption, the photo-carriers have relaxed to the radiative states but they have also populated some dark nonradiative states situated at lower energy. The system is under $\mathrm{CW}$ excitation in a steady state and the recombination of electron-hole pairs is compensated by their photo generation. The results of Fig. 5 simply indicate that the number of populated radiative states is constant when the temperature is raised from $4 \mathrm{~K}$ to $30 \mathrm{~K}$, resulting in a constant PL intensity. When increasing temperature, the carriers initially trapped onto dark states can overcome the energy barrier and populate the radiative states. This leads to the increase of the PL intensity between $30 \mathrm{~K}$ and $50 \mathrm{~K}$. Let us assume that, as discussed above for anatase, further non-radiative states (denoted $\mathrm{b}$ in Fig. 6) with energies even higher than that of the radiative states are present in the samples. Then, at higher temperature, these higher-energy non-radiative states are populated at the expense of the populated radiative states and the PL intensity is quenched for temperatures above $50 \mathrm{~K}$.

In order to simulate this scenario, we consider the four-levels system sketched in Fig. 6 and detailed in the supplementary material. From these states, the lower lying non-radiative states can be populated independently of temperature (in the stationary state, it is not necessary to specify whether the lower-lying dark states are partly populated directly during the relaxation process or via the radiative states). Now electron-hole pairs can either recombine directly from the different states or they can be thermally activated and transferred from the lower lying dark states to the radiative ones or from these to the higher lying non-radiative levels. The radiative recombination is given by

$$
\mathrm{I}(\mathrm{T})=\frac{\mathrm{I}_{0}}{1+\operatorname{Bexp}\left(\frac{-\Delta \mathrm{E}_{\mathrm{b}}}{\mathrm{k}_{\mathrm{B}} \mathrm{T}}\right)+1 /\left(\mathrm{A}_{1}+\mathrm{A}_{2} \exp \left(\frac{-\Delta \mathrm{E}_{\mathrm{D}}}{\mathrm{k}_{\mathrm{B}} \mathrm{T}}\right)\right)} .
$$

Similar to the model that we used for anatase crystals, $\mathrm{B}=\frac{\tau_{\mathrm{R}}}{\tau_{\mathrm{b}}+\tau_{\mathrm{b} \downarrow}}, \mathrm{A}_{1}=\tau_{\mathrm{D} \downarrow} / \tau_{\mathrm{R}}$, and $\mathrm{A}_{2}=\tau_{\mathrm{D}} / \tau_{\mathrm{R}}$. The activation energies are $\Delta E_{i}=\left(E_{i}-E_{R}\right)$, where $E_{R}$ is the energy of the radiative state and $i$ labels the non-radiative states $i=(b, D)$. As shown in Fig. 6, we obtain, as for Eq. (1), a thermally activated process that describes the escape of the carriers toward the level denoted " $b$ " that is characterized by an activation energy $E_{b}$ in the second term of the denominator. But the third term is new, as it described the transfer toward a 
lower lying level denoted "D." At very low temperatures (when $k_{B} T<<\Delta E_{D}$ ), there is no return to the radiative level and only the $A_{1}$ constant that reflects the ratio between the trapping and radiative recombination processes is involved, while at higher temperatures the back transfer to the radiative level is described by the term involving the $\mathrm{A}_{2}$ constant. This level structure allows to explain the shape of the curves in Fig. 5, where photoluminescence is quenched at low temperatures, increases around $50 \mathrm{~K}$, before decreasing slowly again at higher temperatures.

Equation (2) is now used to adjust the results of Fig. 5. Since the PL intensity variation is quite similar for the three samples at low temperatures, we use the same parameter values for $\Delta \mathrm{E}_{\mathrm{D}}$ in the following for their adjustment. The resulting parameter values are summarized in Table II.

The high values of $A_{2}$ and $\Delta E_{D}$ are necessary to reproduce the experimental values by our model but their real value is rather uncertain. Quantitatively, the energetic distribution of the low lying non-radiative states is certainly important in order to describe the thermal activation of the radiative states. Despite the high simplification of our model, we believe that it is qualitatively correct since it reproduces the experimental results.

Concerning the luminescence quenching scenario at high temperatures, we evidenced that it is again only slightly influenced by the orientation of the bulk samples. As given in Table II, only a single high energy non-radiative state is necessary and the PL intensity quenching shows a saturation effect in all three samples.

\section{DISCUSSION}

\section{A. Origin of the broad emission with a large Stokes shift in anatase and rutile}

Figure 1 illustrates the well-known emission features of anatase and rutile, respectively, with a very broad emission band and a very large Stokes shift with respect to absorption.

Photoluminescence spectra of $\mathrm{TiO}_{2}$ have been attributed to different processes where the electron-hole recombination mechanisms show various physical origins such as selftrapped excitons, surface states, or oxygen vacancies. ${ }^{19,32-37}$ Most often, the luminescence band is nevertheless assigned to radiative recombination of self-trapped excitons (STE). ${ }^{34,38}$ We will first look in detail at this mechanism before discussing the relevance of the other ones.

In the formation of STE, ${ }^{39}$ after excitation by a light source, the photo-created electrons and holes relax rapidly down to the minimum-energy states of the conduction band and to the maximum-energy states of the valence band,

TABLE II. Parameters values defined in Eq. (2) for the three rutile $\mathrm{TiO}_{2}$ bulk samples.

\begin{tabular}{lcccrr}
\hline \hline $\begin{array}{l}\mathrm{TiO}_{2} \\
\text { sample }\end{array}$ & $\mathrm{A}_{1}$ & $\mathrm{~A}_{2}$ & $\begin{array}{c}\Delta \mathrm{E}_{\mathrm{D}} \\
(\mathrm{meV})\end{array}$ & $\mathrm{B}$ & \multicolumn{1}{c}{$\begin{array}{c}\Delta \mathrm{E}_{\mathrm{b}} \\
(\mathrm{meV})\end{array}$} \\
\hline $\mathrm{R} 001$ & $0.39 \pm 0.04$ & $48370 \pm 8700$ & $35 \pm 1.0$ & $13 \pm 5.0$ & $43 \pm 6.0$ \\
$\mathrm{R} 110$ & $0.35 \pm 0.05$ & $48370 \pm 8700$ & $35 \pm 1.0$ & $2.3 \pm 0.3$ & $13 \pm 2.6$ \\
$\mathrm{R} 100$ & $0.17 \pm 0.03$ & $48370 \pm 8700$ & $35 \pm 1.0$ & $3.7 \pm 0.4$ & $14.5 \pm 2.2$ \\
\hline \hline
\end{tabular}

respectively. Afterward, due to the strong electron-phonon coupling, another process starts: electrons and holes become dressed with phonons and they build polarons; in other words, the carriers polarize the lattice around them, which is thus strongly distorted.

The Coulomb interaction between electron and hole also has to be taken into account. It results in the formation of excitons that are bound electron-hole pairs. The conjunction of these two effects gives rise to the formation of STEs: those are states where the dipole made by a bound electronhole pair polarizes the lattice. If the induced distortion is strong enough, the exciton can be self-trapped and their effective mass becomes infinite. STEs are thus localized intrinsic states and the observed luminescence in $\mathrm{TiO}_{2}$ is commonly attributed to their recombination.

The features that we observe on the titania emission give valuable information about the STE. First, the line shows a large Stokes shift (around $1.1 \mathrm{eV}$ ) with respect to the absorption edge (Fig. 1) while no specific exciton line is observed in the absorption spectrum. This means that the Coulomb interaction between the electron and the hole is smaller than the carrier-lattice interaction and can be treated in a first approximation as a perturbation. Instead of being strongly bound excitons that are trapped by the deformed lattice, STE in $\mathrm{TiO}_{2}$ are thus pairs of opposite-charge polarons that localize separately and polarize strongly the lattice; they attract further each other in a weaker way through their Coulomb interaction. This scheme is also supported by a second feature of the emission line that has a very large width $(0.6 \mathrm{eV})$. This is hardly compatible with a strongly bound exciton that should show a narrow line. On the contrary, such a broad line cannot be homogenous. The inhomogeneous broadening is a signature of a large variety of levels that can be reached by the carriers. The interacting electron- and hole polarons can explore many different configurations characterized by a broad panel of energies: it results in the observed inhomogeneously broadened line, that is, the superposition of the emission lines of the various configurations.

In Ref. 37, time-resolved PL experiments have been performed. The authors attribute part of the PL emission to the recombination of STEs, which are supposed to be localized on a $\mathrm{TiO}_{6}$ octahedron, which is the building unit of anatase. Another part of the observed PL is attributed to STE emission from distant electron-hole pairs, recombining via a tunneling process. The fact that the observed luminescence shows a very broad emission is rather compatible with the latter idea. Another possibility is that the STE states are extended over several unit cells: Wakabayashi et al. ${ }^{4}$ proposed such spatial delocalization in a lattice plane for exciton states that should have thus a planar structure. A spatially extended STE will thus be sensitive to structural fluctuations in the crystal.

Alternative explanations were however also given to explain the emission in the visible optical spectral range of titania. Zhang et al. concluded that radiative recombination can also be mediated by localized levels within the forbidden gap related to surface defects. ${ }^{33}$ Our present measurements have been performed on bulk crystals. Even if we take into account the weak absorption length of the UV exciting light, which can be estimated to about $200 \mathrm{~nm}$ from published absorption 
coefficients, ${ }^{40}$ the surface-to-volume ratio is strongly reduced when compared to that of nanocrystals. On the other side, the spectral features of the bulk emission do not differ a lot from those of nanocrystals. ${ }^{12}$ Keeping in mind that surface states are present in any condensed matter system and that they can always play a role, it is also straightforward that a bulk monocrystal is the best candidate to observe an intrinsic photoluminescence resulting from a recombination in the volume. Thus, considering the present available experimental elements, we cannot exclude that the observed broad emission occurs from recombination in the crystal volume. It could equally result from an intrinsic feature observed in any structure and geometry like nanocrystals, nanorods, and layers, as in bulk samples.

Besides intrinsic STE, oxygen vacancies as defect states in anatase were also proposed as the origin of the observed PL. This could only explain a small broadening of the PL, but the important width that we observe seems incompatible with such an interpretation. Contrary to what happens in an amorphous material, in the crystalline structure of titanium dioxide, an oxygen vacancy should be in a single configuration, that could only be modified and give rise to a variety of configurations by coupling of vacancies into pairs on neighboring sites. Such defects are thus expected to show discrete lines, without large broadening. On the other hand, as explained below, we will attribute the narrow lines that we observe around $1.5 \mathrm{eV}$ in the rutile sample emission to such STE trapping sites due to oxygen vacancies.

\section{B. Carrier mobility and PL quenching}

Our data about PL efficiency as a function of the temperature allow us now to precise a scheme involving STE made from polarons that can become mobile ${ }^{26,41-42}$ within the titania crystal. The variation of the PL intensity with temperature can indeed be explained as being due to the ability of carriers to be thermally excited and to be spatially dissociated. In a STE, at least one of the carriers (either electron or hole) is in a self-trapped state, which is localized and has been formed by interaction with phonons. These self-trapped states are distributed in a broad energy region around $2.2 \mathrm{eV}$. Similar to the mechanism proposed by Watanabe and Hayashi, ${ }^{37}$ the other carrier is only weakly localized and the distant electron-hole pair can radiatively recombine. Even if such a complex is intrinsic and not related to any defect, its behavior is very similar to extrinsic point defects in crystals that are formed around vacancies or impurities. ${ }^{43}$ In particular, they can experience a diffusive motion within the lattice when they are thermally activated. Such motion of impurities within a crystal is known to be due to hopping and, consequently, to show a migration rate that is thermally activated. For STE in $\mathrm{TiO}_{2}$, at low temperatures, the Coulomb interaction is strong enough to keep a self-trapped electron and a hole at short distances, allowing their radiative recombination. When the temperature increases, hopping of carriers to more distant sites is made possible, reducing strongly the radiative recombination probability. The energies $\Delta \mathrm{E}_{\mathrm{i}}$ in $\mathrm{Eq}$. (1) of our model are thus the activation energies of the respective hopping motion of the two carrier polarons. The most weakly bound carrier, with an energy that lies around $50 \mathrm{meV}$, as given by $\Delta \mathrm{E}_{1}$, dissociates when increasing temperature and transit to distant sites. We further suppose that the different electron-hole pairs, giving rise to PL, are created at low density, in separated spatial regions. Because of this isolation, the electron-hole pairs cannot thermalize within the luminescent states, as it would be the case in bands of delocalized states. Our interpretation of the PL thermal behavior explains the astonishing fact that the whole PL emission band of $600 \mathrm{meV}$ (FWHM) is quenched in a global way when varying temperature from $4 \mathrm{~K}$ to $200 \mathrm{~K}$.

\section{Discrete emission lines in rutile and oxygen vacancies}

The emission spectra of rutile samples as well as their evolution with temperature share some common features with those of anatase, but they differ also by a few points. The spectra show, as in anatase, broad components [Fig. 4(b)] that decrease at high temperature. Here too, they can be attributed to STE made from carrier polarons that can be thermally separated toward distant sites when the temperature increases. Nevertheless, the low temperature behavior, below $50 \mathrm{~K}$, and the set of structured narrow lines are specific of the rutile emission.

We can first explain the origin of the dark states that quench the PL at temperatures below $30 \mathrm{~K}$. Those dark states are located spectrally below the bright states and are thus populated at low temperatures as represented by the dark level of Fig. 6, with an energy $E_{D}=35 \mathrm{meV}$ below the bright level. Both the broad emission and the narrow lines identified in Fig. 4(b) show a common behavior with increasing temperature, as seen in Fig. 4(a). It is thus plausible that the formation of dark states is specific to the rutile crystal structure. The selection rules that would forbid the emission of light should be related to the spin configuration of the carriers that leads to the formation of a dark triplet exciton as the lower lying STE state.

Concerning now the observed set of narrow lines, when it is isolated from the broad components [Fig. 4(c)], it is remarkably similar to the lines observed by Montoncello et $a l .{ }^{44}$ As noted above, the density of oxygen vacancies can be very high in rutile, ${ }^{22}$ we thus tentatively attribute the broad emission band to STE made from "almost free" carrier polarons, while the structured set of narrow lines is due to excitons trapped on oxygen vacancies. Depending on the actual density of defects, the broad emission from almost free carriers can be observable when the number of traps is low enough in the studied samples. The spectral position of the trapped states, which are located at higher energies than the delocalized states, can be explained by the fact that the defects do not cover the whole sample that is thus spatially inhomogeneous. This explains also the fact that the luminescence is not fully quenched at temperatures below $30 \mathrm{~K}$. The lattice distortion is thus different in regions with or without defects and the measured curves are the superpositions of the corresponding spectra. One can notice that, in the sample studied by Montoncello et al., ${ }^{44}$ a broad emission band is not observed as in our samples, but only the 
structured emission due to trapped excitons. That can be explained by a higher density of oxygen vacancies in the former, that shows no regions without defects where STE would be free to move and hop onto any place. As has been shown for Gd-doped $\mathrm{TiO}_{2}$, ${ }^{45}$ density functional theory (DFT) calculations allowing to determine precisely the exact nature of the defect level that are involved in the carrier dynamics and to give their energy position and spin configuration would be very valuable for the understanding energy relaxation properties of titania.

If we consider now the structured emission that we attribute to excitons trapped on defects, we see [Figs. 4(b) and 4(c)] that it can be decomposed into a set of narrow lines that are equally spaced. We can perform here a more sophisticated analysis of the emission that will go beyond the study of Montoncello et al. ${ }^{44}$ who only looked at the spectral width of the whole set of lines. We indeed succeed to fit the spectra that we measured [Fig. 4(c)] using the Franck-Condon model that takes into account the finite temperature and the thermal filling of levels above the lowest lying state. According to this model, initially developed in solids for F-centers, the crystal lattice can be locally distorted by carriers that are trapped on defects or impurities. In the excited state, the lattice equilibrium position is thus spatially shifted. Selection rules are modified and optical transitions can thus involve vibrational states with different quantum numbers in the excited and the ground states. The oscillator strength of the optical transition between the level $m$ in the excited state and the level $n$ in the fundamental state is

$$
F_{n}^{m}=e^{-S} S^{n-m}\left(\frac{m !}{n !}\right)\left(L_{m}^{n-m}(S)\right)^{2} e^{-m \frac{\hbar \omega_{P}}{k_{B} T}}
$$

where $S$ is the Huang-Rhys parameter and $L_{m}^{n-m}$ are the Laguerre polynomials. The optical emission spectrum is the sum of the contribution of all $m$ and $n$ state couples. The thermal occupation of the excited states is taken into account through the Boltzmann occupation function $e^{-m \frac{\hbar \omega_{P}}{k_{B} T}}$ where $\hbar \omega_{P}$ is the phonon energy. This gives rise to the curve with a modulation at the energy $\hbar \omega_{P}$ that is shown in Fig. 4(c). Amazingly, the model fits nicely the observed peaks on the rutile luminescence even if the data are quite complex and the model offers only a few adjustable parameters. This result shows unambiguously the vibrational nature of the levels that give rise to the observed emission with satellite lines. We can also fully quantitatively characterize it: We obtain an energy separation of $\hbar \omega_{P}=14 \mathrm{meV}$ between vibrational levels and a zero-phonon line that is located at $1.546 \mathrm{eV}$. Finally, we can give a value for the Huang-Rhys parameter that is found as large as $S=2.5$. This parameter characterizes the strength of the lattice deformation: its high value shows that the excitons that are trapped on oxygen vacancies polarize strongly, as for the free polarons, the lattice around them and distort it. The observed lines are due to the recombination lines between lattice vibrational states, with a minimum energy that is shifted in the excited electronic state, following the Franck-Condon principle, to account for the lattice deformation.

\section{CONCLUSIONS}

Our analysis of the PL temperature dependence shows that in bulk anatase and rutile $\mathrm{TiO}_{2}$, photo-excited electronhole pairs strongly interact with the lattice by distorting it, giving rise to states which have a high energy inside the band gap and are isolated from each other. These states are intrinsic self-trapped carriers; one of them, forming a polaron, is strongly localized while the second carrier is more weakly bound to the first one. The self-trapped exciton (STE) states that are thus created show a wide range of configurations that explain the broad emission spectral width. When the temperature increases, the hopping transfer of the carriers is thermally activated. Electron and hole can thus migrate separately, leading to a quenching of the radiative recombination. In nanocrystals, this will allow their transfer to surface states where they can be involved in redox reactions. Our measurements and the analysis that we propose show that the main features that are observed in the behavior of nanoparticles are already present in bulk materials. That indicates that the intrinsic properties of titanium dioxide are dominant in the photocarrier dynamics compared to extrinsic processes such as surface trapping. Moreover, we show that carrier-lattice or, in other words, electron-phonon interaction is very important in those intrinsic processes that give rise to both the polarons formation and their trapping.

In rutile $\mathrm{TiO}_{2}$, two different types of luminescent states have been identified at low temperatures around $1.48 \mathrm{eV}$ : Impurity states plausibly related to oxygen vacancies can trap electron-hole pairs, giving rise to sharp PL lines. Those lines are nicely fitted by a Franck-Condon model, showing a distortion of the lattice around the trapped carriers. Their intensity diminishes when increasing the temperature from $4 \mathrm{~K}$ to $130 \mathrm{~K}$. In addition, extended states similar to those of anatase are found in the same spectral region, which form a broad spectral distribution of levels. Their PL emission line is much narrower than in anatase $\mathrm{TiO}_{2}$ and has a width of about $150 \mathrm{meV}$ (FWHM). The states can be populated by electronhole pairs, which are either activated from non-radiative dark states with lower energy or depopulated at higher temperatures via non-radiative states with higher energy.

If we compare the results obtained for anatase and rutile, we see that, in the latter, it deals mainly with the presence of a dark state $35 \mathrm{meV}$ below the emitting state that quenches PL at low temperature, as well as with the role played by the oxygen vacancies. In anatase, the PL behavior when changing the temperature can be fully explained by the dissociation of weakly bound self-trapped excitons where each of the two carriers strongly distorts the lattice. The same result is observed in rutile, but there the carriers can also be trapped on oxygen vacancies. This trapping also gives rise to a spectrally narrow emission at a well-defined energy, with satellites explained in a Franck-Condon model. Both phases show thus a very strong interaction between the photogenerated carriers and the lattice.

\section{SUPPLEMENTARY MATERIAL}

The supplementary material presents a scheme of the multilevel models for radiative and nonradiative states in rutile and anatase titania crystals. 


\section{ACKNOWLEDGMENTS}

The authors gratefully acknowledge the support and funding of the French "Agence Nationale de la Recherche" (ANR) under reference ANR-12-BS10-017.

${ }^{1}$ M. Grätzel, "Photoelectrochemical cells," Nature 414, 338-344 (2001).

${ }^{2} \mathrm{X}$. Chen and S. S. Mao, "Titanium dioxide nanomaterials: Synthesis, properties, modifications, and applications," Chem. Rev. 107, 2891 (2007). ${ }^{3}$ L. Chiodo, J. M. Garcia-Lastra, A. Iacomino, S. Ossicini, J. Zhao, H. Petek, and A. Rubio, "Self-energy and excitonic effects in the electronic and optical properties of $\mathrm{TiO}_{2}$ crystalline phases," Phys. Rev. B 82, 045207 (2010).

${ }^{4}$ K. Wakabayashi, Y. Yamaguchi, T. Sekiya, and S. Kurita, "Time-resolved luminescence spectra in colorless anatase $\mathrm{TiO}_{2}$ single crystal," J. Lumin. 112, 50 (2005)

${ }^{5}$ A. Fujishima, X. T. Zhang, and D. A. Tryk, " $\mathrm{TiO}_{2}$ photocatalysis and related surface phenomena," Surf. Sci. Rep. 63, 515 (2008).

${ }^{6}$ H. Bieber, P. Gilliot, M. Gallart, N. Keller, V. Keller, S. Bégin-Colin, C. Pighini, and N. J. Millot, "Temperature dependent photoluminescence of photocatalytically active titania nanopowders," Catalysis Today 122, 101 (2007).

${ }^{7}$ H. Tang, H. Prasad, R. Sanjinès, P. E. Schmid, and F. Lévy, "Electrical and optical properties of $\mathrm{TiO}_{2}$ anatase thin films," J. Appl. Phys. 75, 2042 (1994).

${ }^{8}$ J. Pascual, J. Camassel, and H. Mathieu, "Fine structure in the intrinsic absorption edge of $\mathrm{TiO}_{2}$, , Phys. Rev. B 18, 5606 (1978).

${ }^{9}$ A. Atmout and R. Leonelli, "Optical properties of rutile near its fundamental band gap,” Phys. Rev. B 51, 6842 (1995).

${ }^{10} \mathrm{C}$. Di Valentin and A. Selloni, "Bulk and surface polarons in photoexcited anatase $\mathrm{TiO}_{2}$," J. Phys. Chem. Lett. 2, 2223 (2011).

${ }^{11} \mathrm{~K}$. N. Glassford and J. R. Chelikowsky, "Structural and electronic properties of titanium dioxide," Phys. Rev. B 46, 1284 (1992).

${ }^{12}$ S. Bégin-Colin, A. Gadalla, G. Le Caër, O. Humbert, F. Thomas, O. Barres, F. Villiéras, L. F. Toma, G. Bertrand, O. Zahraa, M. Gallart, B. Hönerlage, and P. Gilliot, "On the origin of the decay of the photocatalytic activity of $\mathrm{TiO}_{2}$ powders ground at high energy," J. Phys. Chem. C 113, 16589 (2009).

${ }^{13}$ J.-M. Herrmann, "Heterogeneous photocatalysis: Fundamentals and applications to the removal of various types of aqueous pollutants," Catalysis Today 53, 115 (1999)

${ }^{14} \mathrm{Q}$. Zhang, L. Gao, and J. Guo, "Effects of calcination on the photocatalytic properties of nanosized $\mathrm{TiO}_{2}$ powders prepared by $\mathrm{TiCl}_{4}$ hydrolysis," Appl. Catalysis 26, 207 (2000).

${ }^{15}$ Y. Yamada and Y. Kanemitsu, "Blue photoluminescence of highly photoexcited rutile $\mathrm{TiO}_{2}$ : Nearly degenerate conduction-band effects," Phys. Rev. B 82, 113103 (2010).

${ }^{16}$ Y. Yamada and Y. Kanemitsu, "Determination of electron and hole lifetimes of rutile and anatase $\mathrm{TiO}_{2}$ single crystals," Appl. Phys. Lett. 101, 133907 (2012).

${ }^{17}$ A. Stevanovic and J. T. Yates, "Electron hopping through $\mathrm{TiO}_{2}$ powder: A study by photoluminescence spectroscopy," J. Phys. Chem. C 117(46), 24189-24195, (2013)

${ }^{18}$ N. A. Deskins and M. Dupuis, "Electron transport via polaron hopping in bulk $\mathrm{TiO}_{2}$ : A density functional theory characterization,” Phys. Rev. B 75, 195212 (2007)

${ }^{19}$ M. Watanabe, H. Yagasaki, S. Sasaki, and T. Hayashi, "Luminescence process in anatase $\mathrm{TiO}_{2}$ studied by time-resolved spectroscopy," Int. J. Mod. Phys. B 15, 3997 (2001).

${ }^{20}$ M. Watanabe, S. Sasaki, and T. Hayashi, "Time-resolved study of photoluminescence in anatase $\mathrm{TiO}_{2}$," J. Lumin. 87-89, 1234 (2000).

${ }^{21}$ K. Song and R. T. Williams, Self-Trapped Excitons (Springer, 1993).

${ }^{22}$ P. Deak, B. Aradi, and T. Frauenhrim, "Quantitative theory of the oxygen vacancy and carrier self-trapping in bulk $\mathrm{TiO}_{2}$," Phys. Rev. B 86, 195206 (2012).
${ }^{23}$ J. Zhang, X. Chen, Y. Shen, Y. Li, Y. Hu, and J. Chu, "Synthesis, surface morphology, and photoluminescence properties of anatase iron-doped titanium dioxide nano-crystalline films," Phys. Chem. Chem. Phys. 13, 13096-13105 (2011).

${ }^{24}$ S. M. Gupta and M. Trpathi, "A review of $\mathrm{TiO}_{2}$ nanoparticles," Chin. Sci. Bull. 56, 1639-1657 (2011).

${ }^{25}$ M. Kapilashrami, Y. Zhang, Y.-S. Liu, A. Hagfeldt, and J. Guo, "Probing the optical properties and electronic struture of $\mathrm{TiO}_{2}$ nanomaterials for renewable energy applications," Chem. Rev. 114, 9662-9707 (2014).

${ }^{26}$ J. Schneider, M. Matsuoka, M. Takeuchi, J. Zhang, Y. Horiuchi, M. Anpo, and D. W. Bahnemann, "Understanding $\mathrm{TiO}_{2}$ photocatalysis: Mechanisms and materials," Chem. Rev. 114(19), 9919-9986 (2014).

${ }^{27}$ L. Schlur, S. Begin-Colin, P. Gilliot, M. Gallart, G. Carre, S. Zafeiratos, N. Keller, V. Keller, P. Andre, J.-M. Greneche, B. Hezard, M. H. Desmonts, and G. Pourroy, "Effect of ball-milling and Fe-/Al-doping on the structural aspect and visible light photocatalytic activity of $\mathrm{TiO}_{2}$ towards Escherichia coli bacteria abatement," Mater. Sci. Eng. C 38, 11-19 (2014).

${ }^{28}$ S. Shionoya, T. Koda, and A. Era, "Nature of luminescence transitions in ZnS crystals," J. Phys. Soc. Jpn 19, 1157 (1964).

${ }^{29}$ J. S. Prener and D. J. Weil, "The luminescent center in self-activated $\mathrm{ZnS}$ phosphors," J. Electrochem. Soc. 106, 409 (1959).

${ }^{30}$ S. Monticone, R. Tufeu, A. V. Kanaev, E. Scolan, and C. Sanchez, "Quantum size effect in $\mathrm{TiO}_{2}$ nanoparticles: Does it exist?," Appl. Surf. Sci. 162-163, 565 (2000).

${ }^{31}$ X. Wang, Z. Feng, J. Shi, G. Jia, S. Shen, J. Zhou, and C. Li, "Trap states and carrier dynamics of $\mathrm{TiO}_{2}$ studied by photoluminescence spectroscopy under weak excitation condition," Phys. Chem. Chem. Phys. 12, 7083 (2010).

${ }^{32}$ M. Scepanovic, Z. D. Dohcevic-Mitrovic, and I. Hinic, "Photoluminescence of laser-synthesized anatase titanium dioxide nanopowders," Mater. Sci. Forum 494, 265 (2005).

${ }^{33}$ W. F. Zhang, M. S. Zhang, Z. Yin, and Q. Chen, "Photoluminescence in anatase titanium dioxide nanocrystals,” Appl. Phys. B 70, 261 (2000).

${ }^{34} \mathrm{H}$. Tang, H. Berger, P. E. Schmid, F. Lévy, and G. Burri, "Photoluminescence in $\mathrm{TiO}_{2}$ anatase single crystals," Solid State Commun. 87, 847 (1993).

${ }^{35}$ T. Sekiya, S. Kamei, and S. J. Kurita, "Luminescence of anatase $\mathrm{TiO}_{2}$ single crystals annealed in oxygen atmosphere," J. Lumin. 87-89, 1140 (2000).

${ }^{36}$ K. Y. Jung, S. B. Park, and M. J. Anpo, "Photoluminescence and photoactivity of titania particles prepared by the sol-gel technique: Effect of calcination temperature," Photochem. Photobiol. A. 170, 247 (2005).

${ }^{37}$ M. Watanabe and T. Hayashi, "Time-resolved study of self-trapped exciton luminescence in anatase $\mathrm{TiO}_{2}$ under two-photon excitation," J. Lumin. 112, 88 (2005).

${ }^{38}$ N. Hosaka, T. Sekiya, and S. Kurita, "Excitonic state in anatase $\mathrm{TiO}_{2}$ single crystal," J. Lumin. 72-74, 874 (1997).

${ }^{39}$ R. T. Williams and K. S. Song, "The self-trapped exciton," J. Phys. Chem. Solids 51(7), 679-716 (1990).

${ }^{40}$ O. Madelung, U. Rössler, and M. Schulz, "Landolt-Börnstein III-41D," Non-Tetrahedrally Bonded Binary Compounds II (Springer, 2000).

${ }^{41}$ L. J. Antila, F. G. Santomauro, L. Hammarström, D. L. A. Fernandesa, and J. Sá, "Hunting for the elusive shallow traps in $\mathrm{TiO}_{2}$ anatase," Chem. Commun. 51, 10914 (2015).

${ }^{42}$ A. M. Stoneham, J. Gavartin, A. L. Shluger, A. V. Kimmel, D. Muñoz Ramo, H. M. Rønnow, G. Aeppli, and C. Renner, "Trapping, self-trapping and the polaron family," J. Phys. Condens. Matter 19, 255208 (2007).

${ }^{43}$ H. Mehere, Diffusion in Solids (Springer, 2007)

${ }^{44}$ F. Montoncello, M. C. Carotta, B. Cavicchi, M. Ferroni, A. Giberti, V. Guidi, C. Malagù, G. Martinelli, and F. Meinardi, "Near-infrared photoluminescence in titania: Evidence for phonon-replica effect," J. Appl. Phys. 94, 1501 (2003)

${ }^{45}$ R. Imani, R. Dillert, D. W. Bahnemann, M. Pazoki, T. Apih, V. Kononenko, N. Repar, V. Kralj-Iglič, G. Boschloo, D. Drobne, T. Edvinsson, and A. Iglič, "Multifunctional gadolinium-doped mesoporous $\mathrm{TiO}_{2}$ nanobeads: Photoluminescence, enhanced spin relaxation, and reactive oxygen species photogeneration, beneficial for cancer diagnosis and treatment," Small 13(20), 1700349 (2017). 